\title{
The comparative study on analytical solutions and numerical solutions of the deformation of a circular hole in layered rock mass
}

\author{
Zhizeng Zhang ${ }^{1, a}$, Xiaochang $\mathrm{Li}^{1, \mathrm{~b}}$ and Xin Zhang ${ }^{1, \mathrm{c}}$ \\ ${ }^{1}$ School of Civil Engineering and Architecture, Zhongyuan University of Technology, Zhengzhou, \\ 450007 China

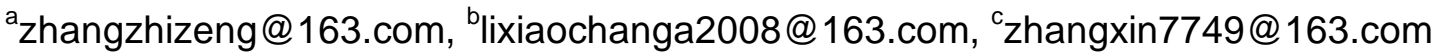

Keywords: Layered rock mass; approximate analytical solution; numerical solution; applicability. Abstract. This paper derived an approximate deformation analytical solution of a circular hole which is parallel to the isotropic plane of the layered rock mass. The data comparisons of approximate solutions and numerical solutions are conducted in different radii and stress conditions through the FLAC3D software. The accuracy and applicability of the approximate solution are proved by the comparisons of the data and the analyses of error.

\section{Introduction}

It is a structure characteristic of the layered rock mass that there distributes a set of structural planes with absolute advantages. The existence of these structure planes makes the deformation and strength of the layered rock mass display anisotropy, which makes the mechanics problems more complicated. The layered rock mass can be generally treated as transverse isotropic in mechanics.

There have been some studies about holes deformation in layered rock mass. Lekhnitskii solved a displacement analytical solution for a circular hole in layered rock mass under axisymmetric stress condition[1]. Zhang deduced displacement analytical solutions for tunnels with both circular and arbitrary cross section in layered rock mass under two unequal normal stresses conditions[2,3]. However, they both presumed that the hole direction is perpendicular to the isotropic plane of the layered rock mass. There are few studies concerned with the deformation solutions of the holes parallel to the isotropic plane.

The circular hole parallel to the isotropic plane in the layered rock mass belongs to the category of the orthogonal anisotropy. Han gave both the physical equations for plane strain problem in polar coordinates and the approximate stress analytical solutions of a circular hole in orthogonal anisotropic rock mass[4,5]. According to Han, this paper derived an approximate deformation analytical solution of a circular hole parallel to the isotropic plane of the layered rock mass. And the numerical analysis software FLAC3D is used to compare the data of the approximate solutions and the numerical solutions. The accuracy and applicability of the approximate solution are verified.

\section{The approximate deformation analytical solution}

Model assumptions. The assumptions for the circular hole are presented firstly. The following assumptions are used:

(1) The layered rock mass is continuous, homogeneous and linear elastic, and the strain and deformation are tiny;

(2) The direction of the circular hole is parallel to the isotropic plane of the layered rock mass, and $a$ is the radius;

(3) The hole length is much bigger than $a$. It is a plane strain problem;

(4) The horizontal stress is $q$ and the vertical stress is $p$;

(5) The gravity of the layered rock mass is much smaller than the initial stress, therefore the gravity can be neglected in computation.

Thus an ideal model of the circular hole is set up, as is shown in Fig. 1. 


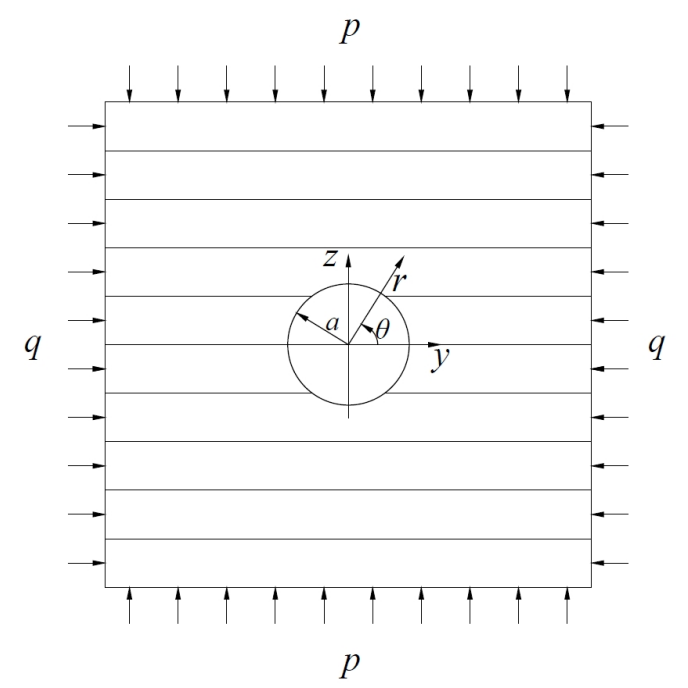

Fig. 1 The ideal model of the circular hole

As the model shows in Fig. 1, xoy plane is the isotropic plane of the layered rock and $o z$ is the symmetry axis. The parameters of layered rock are as follows: $E, \mu$ and $G$ are respectively elastic modulus, Poisson's ratio and shear modulus in the isotropic plane; $E^{\prime}, \mu^{\prime}$ and $G^{\prime}$ are that in the direction of symmetry axis.

The approximate deformation analytical solution. On the basis of the physical equations and approximate stress analytical solutions given by Han[4,5], this paper obtained the approximate deformation analytical solution for the hole shown in Fig. 1 as:

$$
\begin{aligned}
u_{r}= & \frac{p+q}{2} \frac{a^{2}}{r}\left(N_{11}-N_{12}\right)+\frac{p-q}{2}\left[\frac{(1-2 A) a^{4}}{r^{3}}\left(N_{11}-N_{12}\right)+\frac{2(A-2) a^{2}}{r} N_{11}+\frac{1}{5} \frac{A a^{6}}{r^{5}}\left(4 N_{11}-10 N_{12}\right)\right] \cos 2 \theta \\
& +\frac{p-q}{2}\left[\frac{(A-2) a^{2}}{r} N_{13}+\frac{(1-2 A) a^{4}}{r^{3}} N_{13}+\frac{A a^{6}}{r^{5}} N_{13}\right] \sin 2 \theta
\end{aligned}
$$

(1)

Where, $u_{r}$ is the radial deformation. $a, r$, and $\theta$ are respectively the radius of the circular hole, the radius and the angle in polar coordinates. The other parameters in Eq. (1) are respectively:

$$
\begin{aligned}
A= & -\frac{5\left[\frac{1-\mu^{2}}{E}+\frac{1+2 \mu^{\prime}(1+\mu)}{E^{\prime}}-\frac{E \mu^{\prime 2}}{E^{\prime 2}}\right]}{\frac{7\left(1-\mu^{2}\right)}{E}+\frac{7-2 \mu^{\prime}(1+\mu)}{E^{\prime}}-\frac{7 E \mu^{\prime 2}}{E^{\prime 2}}+\frac{1}{G^{\prime}}} \\
N_{11}= & \frac{1}{8}\left[\frac{3\left(1-\mu^{2}\right)}{E}+\frac{3-2 \mu^{\prime}(1+\mu)}{E^{\prime}}-\frac{3 E \mu^{\prime 2}}{E^{\prime 2}}+\frac{1}{G^{\prime}}\right]+\frac{1}{2}\left(\frac{1-\mu^{2}}{E}-\frac{1}{E^{\prime}}+\frac{E \mu^{\prime 2}}{E^{\prime 2}}\right) \cos 2 \theta \\
& +\frac{1}{8}\left[\frac{1-\mu^{2}}{E}+\frac{1+2 \mu^{\prime}(1+\mu)}{E^{\prime}}-\frac{E \mu^{\prime 2}}{E^{\prime 2}}-\frac{1}{G^{\prime}}\right] \cos 4 \theta \\
N_{12}= & \frac{1}{8}\left[\frac{1-\mu^{2}}{E}+\frac{1-6 \mu^{\prime}(1+\mu)}{E^{\prime}}-\frac{E \mu^{\prime 2}}{E^{\prime 2}}-\frac{1}{G^{\prime}}\right]-\frac{1}{8}\left[\frac{1-\mu^{2}}{E}+\frac{1+2 \mu^{\prime}(1+\mu)}{E^{\prime}}-\frac{E \mu^{\prime 2}}{E^{\prime 2}}-\frac{1}{G^{\prime}}\right] \cos 4 \theta \\
N_{13}= & -\frac{1}{2}\left(\frac{1-\mu^{2}}{E}-\frac{1}{E^{\prime}}+\frac{E \mu^{\prime 2}}{E^{\prime 2}}\right) \sin 2 \theta-\frac{1}{4}\left[\frac{1-\mu^{2}}{E}+\frac{1+2 \mu^{\prime}(1+\mu)}{E^{\prime}}-\frac{E \mu^{\prime 2}}{E^{\prime 2}}-\frac{1}{G^{\prime}}\right] \sin 4 \theta
\end{aligned}
$$

Eq. (1) is a very complicated analytical solution, and the data comparison method is used in the following part in order to verify its availability.

\section{Data comparisons and analyses}

Calculation model. FLAC3D is used in this part and Fig. 2 shows the division of computation mesh, and the size of the model is $50 \mathrm{~m} \times 50 \mathrm{~m}$, and the hole radius is $2 \mathrm{~m}$. The boundary conditions of the model 
are as follows: the left and right side of the model is restricted in the horizontal direction, and the top and bottom of the model is restricted in the vertical direction. The parameters of the layered rock are selected as follows[6]: $E=35.33 \mathrm{Gpa}, \mu=0.286, E^{\prime}=24.7 \mathrm{Gpa}, \mu^{\prime}=0.219, G^{\prime}=12.09 \mathrm{Gpa}$.

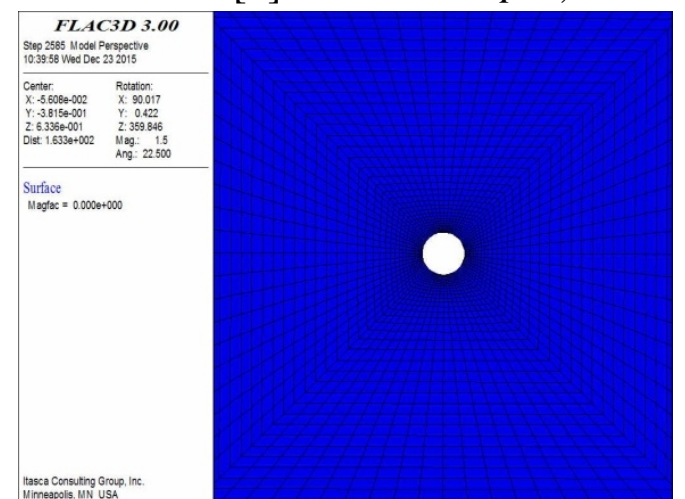

Fig. 2 The division of computation mesh

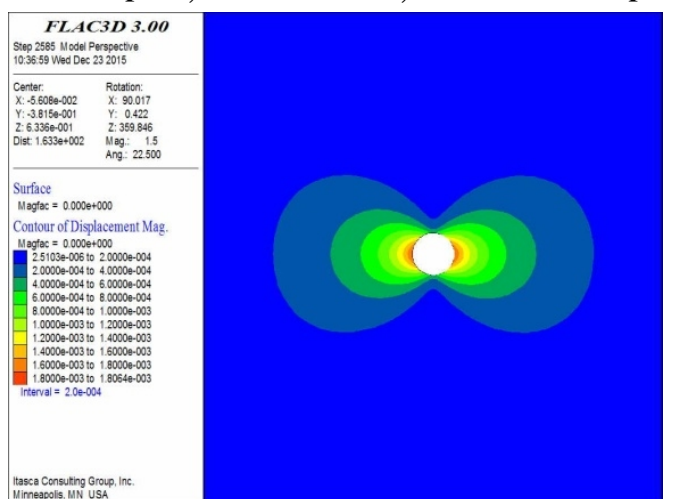

Fig. 3 The radial deformation nephogram

The data comparisons and analyses for the case of $p=10 \mathrm{Mpa}, q=20 \mathrm{Mpa}$. Stress data are selected as $p=10 \mathrm{Mpa}, q=20 \mathrm{Mpa}$, Fig. 3 shows the radial deformation nephogram, which shows that the deformation is symmetrical. In order to verify whether the approximate solution meet the symmetry, the data of two groups of points on the hole wall $(r=2 m)$ are selected to be compared firstly, and the angle variation range of which are $\theta=0^{\circ} \square 180^{\circ}$ and $\theta=-90^{\circ} \square 90^{\circ}$, the location of the points selected and the data results are shown in Table 1 and Table 2 respectively. Data comparison curves are shown in Fig. 4 and Fig. 5.

Note: In the tables and the curves, for the Numerical Solution and Analytical Solution, the positive number means the deformation points the center of the hole, the negative number means the deformation deviates from the center.

Table 1 The comparison results of the analytical and numerical solutions ( $r=2 m, \theta=0^{\circ} \square 180^{\circ}$ )

\begin{tabular}{cccccc}
\hline $\begin{array}{c}\text { Point } \\
\text { number }\end{array}$ & $\begin{array}{c}\text { Polar } \\
\text { Coordinates }\end{array}$ & $\begin{array}{c}\text { Numerical } \\
\text { Solution }(\mathrm{cm})\end{array}$ & $\begin{array}{c}\text { Analytical } \\
\text { Solution }(\mathrm{cm})\end{array}$ & $\begin{array}{c}\text { Absolute } \\
\text { Error(cm) }\end{array}$ & $\begin{array}{c}\text { Relative } \\
\text { Error(\%) }\end{array}$ \\
\hline 1 & $(2,0)$ & 0.1805 & 0.1965 & 0.0160 & 8.84 \\
2 & $(2,10)$ & 0.1771 & 0.1932 & 0.0161 & 9.09 \\
3 & $(2,20)$ & 0.1674 & 0.1834 & 0.0161 & 9.61 \\
4 & $(2,30)$ & 0.1482 & 0.1670 & 0.0188 & 12.68 \\
5 & $(2,45)$ & 0.1189 & 0.1299 & 0.0110 & 9.26 \\
6 & $(2,60)$ & 0.0895 & 0.0820 & -0.0075 & 8.35 \\
7 & $(2,75)$ & 0.0645 & 0.0381 & -0.0265 & 41.03 \\
8 & $(2,90)$ & 0.0570 & 0.0198 & -0.0372 & 65.26 \\
9 & $(2,105)$ & 0.0646 & 0.0381 & -0.0265 & 41.05 \\
10 & $(2,120)$ & 0.0895 & 0.0820 & -0.0075 & 8.43 \\
11 & $(2,135)$ & 0.1190 & 0.1299 & 0.0109 & 9.16 \\
12 & $(2,150)$ & 0.1483 & 0.1670 & 0.0187 & 12.59 \\
13 & $(2,160)$ & 0.1675 & 0.1834 & 0.0160 & 9.52 \\
14 & $(2,170)$ & 0.1771 & 0.1932 & 0.0161 & 9.09 \\
15 & $(2,180)$ & 0.1806 & 0.1965 & 0.0159 & 8.79 \\
\hline
\end{tabular}


Table 2 The comparison results of the analytical and numerical solutions ( $r=2 m, \theta=-90^{\circ} \square 90^{\circ}$ )

\begin{tabular}{cccccc}
\hline $\begin{array}{c}\text { Point } \\
\text { number }\end{array}$ & $\begin{array}{c}\text { Polar } \\
\text { Coordinates }\end{array}$ & $\begin{array}{c}\text { Numerical } \\
\text { Solution }(\mathrm{cm})\end{array}$ & $\begin{array}{c}\text { Analytical } \\
\text { Solution }(\mathrm{cm})\end{array}$ & $\begin{array}{c}\text { Absolute } \\
\text { Error }(\mathrm{cm})\end{array}$ & $\begin{array}{c}\text { Relative } \\
\text { Error(\%) }\end{array}$ \\
\hline 1 & $(2,-90)$ & 0.0571 & 0.0198 & -0.0373 & 65.30 \\
2 & $(2,-75)$ & 0.0646 & 0.0381 & -0.0266 & 41.10 \\
3 & $(2,-60)$ & 0.0895 & 0.0820 & -0.0076 & 8.44 \\
4 & $(2,-45)$ & 0.1190 & 0.1299 & 0.0109 & 9.18 \\
5 & $(2,-30)$ & 0.1483 & 0.1670 & 0.0187 & 12.64 \\
6 & $(2,-20)$ & 0.1674 & 0.1834 & 0.0160 & 9.58 \\
7 & $(2,-10)$ & 0.1772 & 0.1932 & 0.0161 & 9.08 \\
8 & $(2,0)$ & 0.1805 & 0.1965 & 0.0160 & 8.84 \\
9 & $(2,10)$ & 0.1771 & 0.1932 & 0.0161 & 9.09 \\
10 & $(2,20)$ & 0.1674 & 0.1834 & 0.0161 & 9.61 \\
11 & $(2,30)$ & 0.1482 & 0.1670 & 0.0188 & 12.68 \\
12 & $(2,45)$ & 0.1189 & 0.1299 & 0.0110 & 9.26 \\
13 & $(2,60)$ & 0.0895 & 0.0820 & -0.0075 & 8.35 \\
14 & $(2,75)$ & 0.0645 & 0.0381 & -0.0265 & 41.03 \\
15 & $(2,90)$ & 0.0570 & 0.0198 & -0.0372 & 65.26 \\
\hline
\end{tabular}

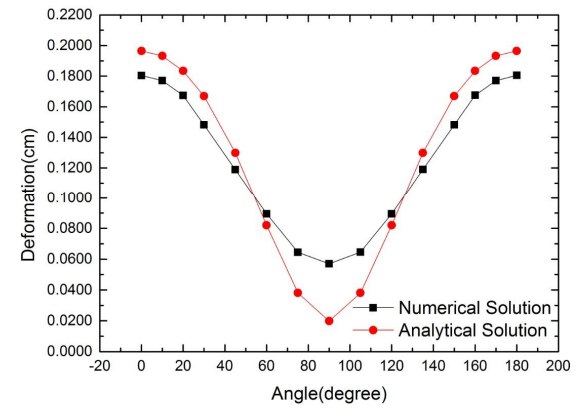

Fig. 4 The data comparison curves $\left(r=2 m, \theta=0^{\circ} \square 180^{\circ}\right)$

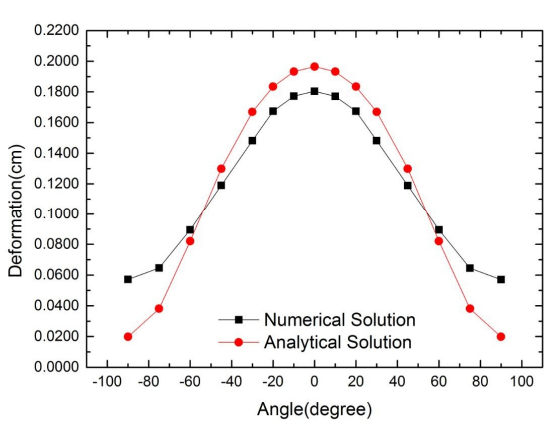

Fig. 5 The data comparison curves

$$
\left(r=2 m, \theta=-90^{\circ} \square 90^{\circ}\right)
$$

The above comparisons of the two groups of data show that the change tendency of approximate solutions is in accordance with that of the numerical solutions, also with symmetry, but more volatility, which indicates that the approximate solutions basically reflect the actual material deformation law. Therefore, data analyses could be conducted in 1/4 area only. The figures show that, within the limits of $\theta=0^{\circ} \square 90^{\circ}$, as the angle increase, the errors of the approximate solutions and numerical solutions hold steady firstly, then decrease gradually until the two curves intersect. After that the errors increased greatly, and the maximum error is at $\theta=90^{\circ}$. The intersection point of the curves is among the range of $\theta=45^{\circ} \square 60^{\circ}$, which suggests that at some point among $\theta=45^{\circ} \square 60^{\circ}$, the approximate solution can achieve precise value.

In order to test the applicability of the approximate solutions in the region away from the hole wall, some points are selected by raising the radius gradually. Through the comparisons, it can be found that the errors increase with the raises of radius, but the curves keep the same trend as before. Fig. 6 and Fig. 7 respectively show the data comparison results for $r=5 m$ and $r=8 m$, which show that the two curves still intersect among $\theta=45^{\circ} \square 60^{\circ}$. 


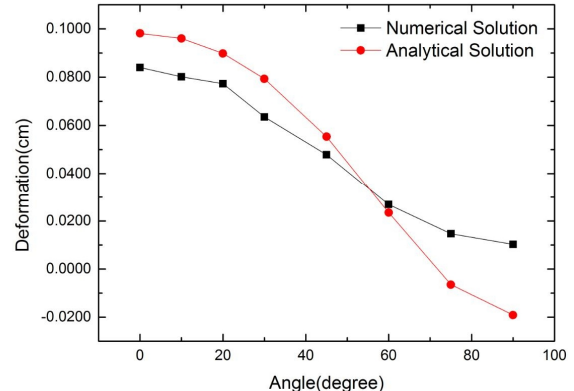

Fig. 6 The data comparison curves $\left(r=5 m, \theta=0^{\circ} \square 90^{\circ}\right)$

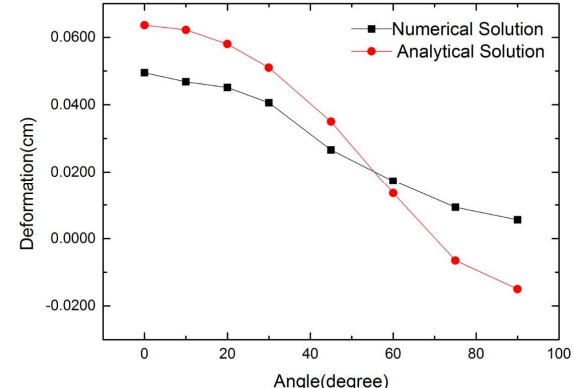

Fig. 7 The data comparison curves

$$
\left(r=8 m, \theta=0^{\circ} \square 90^{\circ}\right)
$$

Through the above analyses, we can infer that the results of the approximate solutions have the same change tendency as the numerical solutions, just more volatility. And the errors may gradually increase with the raises of the radius, but the position of the curve intersection basically remain unchanged among $\theta=45^{\circ} \square 60^{\circ}$, which shows the best applicable scope of the approximate solution is $\theta=45^{\circ} \square 60^{\circ}$.

The data comparisons and analyses as the change of the horizontal stress. The data comparisons are conducted in different stress conditions. The vertical stress remains constant ( $p=10 M p a)$ while the horizontal stress gradually increases from $q=20 \mathrm{Mpa}$ to $q=80 \mathrm{Mpa}$. Data comparison results are shown in Fig. 8 to Fig. 11 for the case of $q=30 M p a$, and Fig. 12 to Fig. 15 are that for the case of $q=80 \mathrm{Mpa}$.

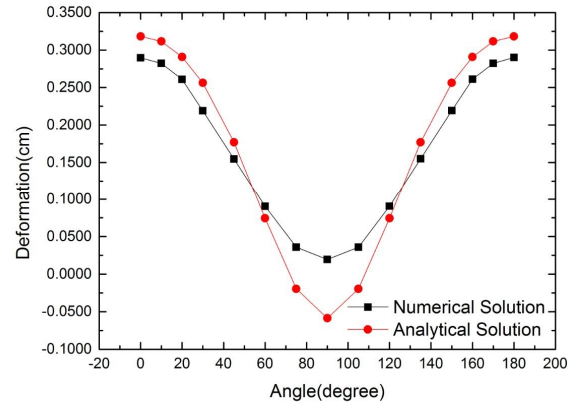

Fig. 8 The data comparison curves $\left(q=30 M p a, r=2 m, \theta=0^{\circ} \square 180^{\circ}\right)$

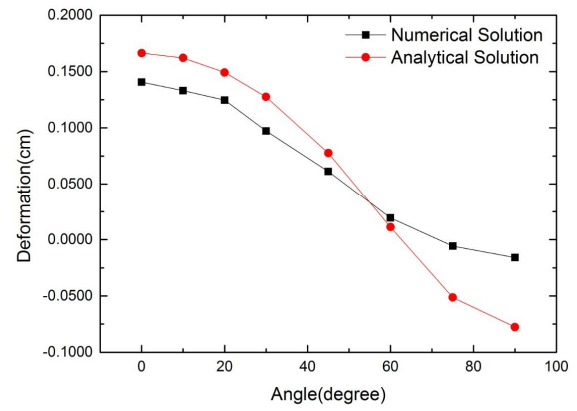

Fig. 10 The data comparison curves $\left(q=30 \mathrm{Mpa}, r=5 \mathrm{~m}, \theta=0^{\circ} \square 90^{\circ}\right)$

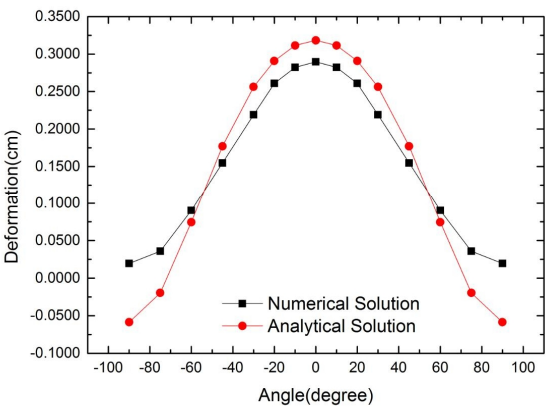

Fig. 9 The data comparison curves $\left(q=30 M p a, r=2 m, \theta=-90^{\circ} \square 90^{\circ}\right)$

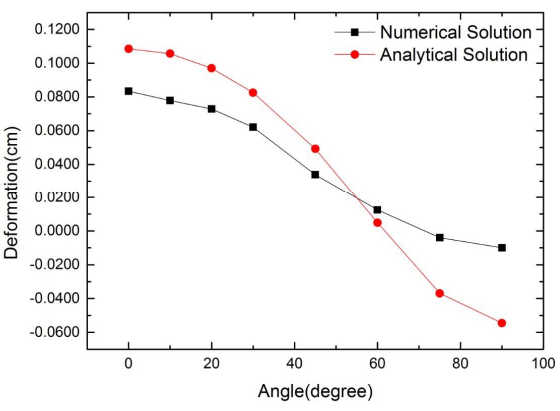

Fig. 11 The data comparison curves $\left(q=30 \mathrm{Mpa}, r=8 m, \theta=0^{\circ} \square 90^{\circ}\right)$ 


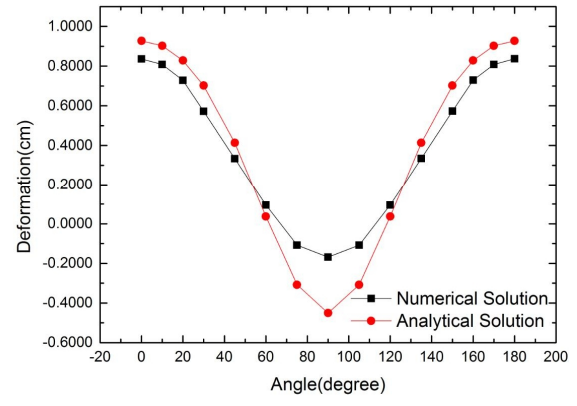

Fig. 12 The data comparison curves $\left(q=80 \mathrm{Mpa}, r=2 m, \theta=0^{\circ} \square 180^{\circ}\right)$

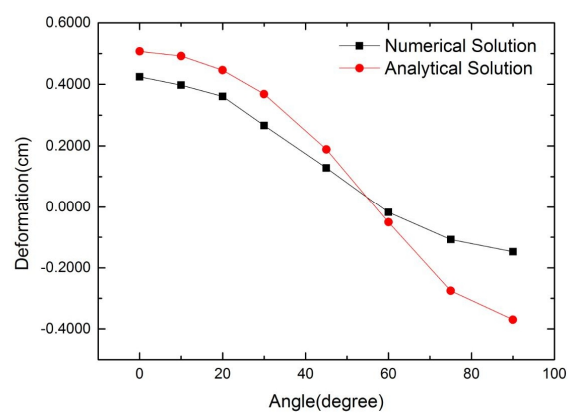

Fig. 14 The data comparison curves $\left(q=80 \mathrm{Mpa}, r=5 \mathrm{~m}, \theta=0^{\circ} \square 90^{\circ}\right)$

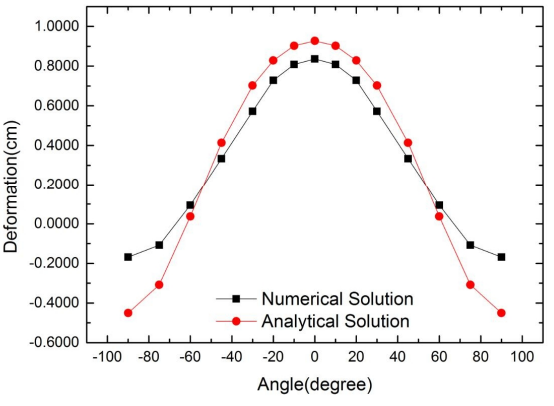

Fig. 13 The data comparison curves $\left(q=80 \mathrm{Mpa}, r=2 m, \theta=-90^{\circ} \square 90^{\circ}\right)$

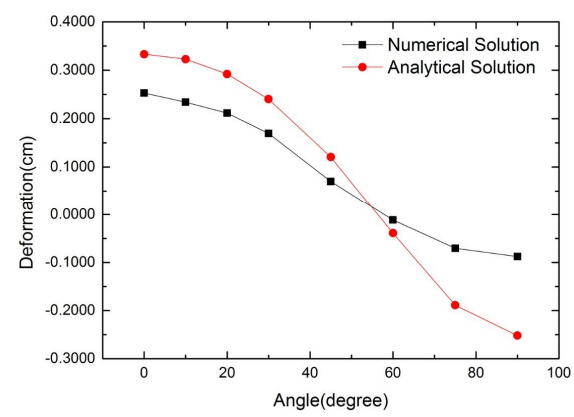

Fig. 15 The data comparison curves $\left(q=80 \mathrm{Mpa}, r=8 m, \theta=0^{\circ} \square 90^{\circ}\right)$

The curves of the two kinds of stress conditions keep the same trend with before, which show that the comparison results are not affected by the change of stress conditions. For every two curves, the position of the intersection is almost the same, they all among the range of $\theta=45^{\circ} \square 60^{\circ}$, which means that within this scope, approximate solution has high precision.

\section{Conclusions}

The main conclusions are summarized as follows:

(1) The approximate deformation analytical solution of a circular hole parallel to the isotropic plane of the layered rock mass is derived;

(2) The data comparisons and analyses show that the approximate solutions basically reflect the actual materials deformation law. Within the limits of $\theta=0^{\circ} \square 90^{\circ}$, as the angle increases, the errors of the approximate solutions and numerical solutions hold steady firstly, then decrease gradually until the two curves intersect. After that the errors increase greatly, and the maximum error is at $\theta=90^{\circ}$;

(3) Within the limits of $\theta=45^{\circ} \square 60^{\circ}$, the curves of approximate solutions and numerical solutions intersect, the error is relatively small, which is the best applicable scope of the approximate solution, and do not change with the raises of the radius;

(4) The errors may gradually increase with the raises of the radius, and the change of the stress conditions does not affect the changing rules and the application scope of the approximate solution.

\section{Acknowledgements}

This research is supported by the Joint Fund for Fostering Talents of National Natural Science Foundation of China and Henan province (No. U1204509).

\section{References}

[1] S.G. Lekhniskii: Theory of elasticity of an anisotropic body, Mir Publishers, Moscow(1981), in press. 
[2] Z.Z. Zhang, Z.K. Li and M.G. Xu: Gold. Vol. 31(2010), p. 23

[3] Z. Zhang, Y. Sun: Int. J. Rock Mech. Min. Sci. Vol. 48(2011), p. 1359

[4] C.R. Han, S.W. Bai and B. Zhang. Rock Soil Mech. Vol. 28(2007), p. 2249

[5] C.R. Han, S.W. Bai and B Zhang: Rock Soil Mech. Vol. 28(2007), p. 2593

[6] Y.S. Liu, H.L. Fu and Y.M. Wu, et al: J. Cent. South U. (Sci. Technol.) Vol. 44(2013), p. 3398 